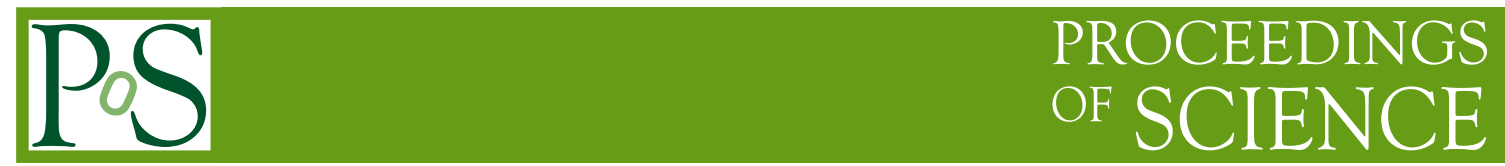

\title{
Chemical Abundances in Globular Clusters
}

\section{David Yong*}

Australian National University

E-mail: yongemso.anu.edu.au

The Milky Way globular clusters are ideal laboratories for testing the predictions of nuclear astrophysics. Driven by Hubble Space Telescope photometry and high-resolution multi-object spectrographs on 8m-class telescopes, the study of chemical abundances in globular clusters is a vibrant area of astronomical research. These studies are providing new insights into stellar evolution, stellar nucleosynthesis and nuclear astrophysics. These data are also presenting new challenges to stellar nucleosynthesis and the chemical evolution of these ancient stellar clusters.

XII International Symposium on Nuclei in the Cosmos,

August 5-12, 2012

Cairns, Australia

${ }^{*}$ Speaker. 


\section{Introduction}

The globular clusters of the Milky Way have played a major role in modern astronomy. They have long been regarded to be the best example of a simple stellar population, i.e., a coeval and chemically homogeneous population [1]. These simple stellar populations are the oldest objects for which reliable ages can be obtained. Prior to 2003, the ages of globular clusters as inferred from isochrone fitting [2] provided firm lower limits to the age of the Universe (see Figure 1). After 2003, the ages inferred from globular clusters provide an important independent check on the WMAP results [3].

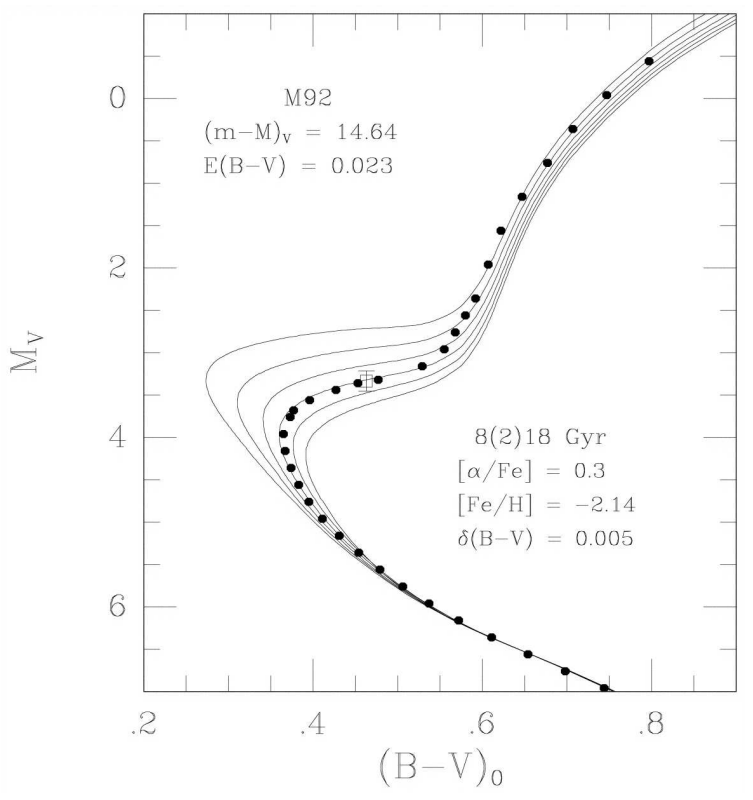

Figure 1: Isochrone fitting to the globular cluster M92. (Taken from Figure 3, A Distance-Independent Age for the Globular Cluster M92, Grundahl et al., The Astronomical Journal, Volume 120, Issue 4, 2000, pp. 1884-1891 [2]. Reproduced by permission of the AAS.)

In the context of the Nuclei in the Cosmos meetings, globular clusters are ideal laboratories for testing the predictions of nuclear astrophysics. The brightness, colours and chemical abundances of individual stars are the direct result of stellar evolution and stellar nucleosynthesis within a given object. Recent results demonstrate, however, that every well studied Galactic globular cluster exhibits large star-to-star abundance variations for the light elements $\mathrm{O}$ and $\mathrm{Na}$, and thus, globular clusters cannot be regarded to be simple stellar populations. Furthermore, a small but growing number of clusters also show variations in Fe-peak elements. (Reviews on Galactic globular clusters include [4], [5], and [6], and some excellent material prepared by Jay Anderson can be found at the following websites ${ }^{123}$.)

\footnotetext{
${ }^{1}$ http://hubblesite.org/newscenter/archive/releases/2010/28/video/d/

${ }^{2}$ http://hubblesite.org/pubinfo/ppt/2010/28/ppt.ppt

${ }^{3} \mathrm{http} / / /$ hubblesite.org/newscenter/archive/releases/2010/28/video/b/
} 


\section{Chemical Abundances and Nucleosynthesis}

In this section, I offer five examples of how chemical abundance measurements in globular clusters have provided valuable new insights into stellar evolution, stellar nucleosynthesis and nuclear astrophysics. The examples are presented in order of atomic number of the species being investigated.

$i$. Measurements of lithium in the metal-poor globular cluster NGC 6397 have provided strong observational constraints on first dredge-up, atomic diffusion and turbulent mixing [7]. (See Figure 2.)

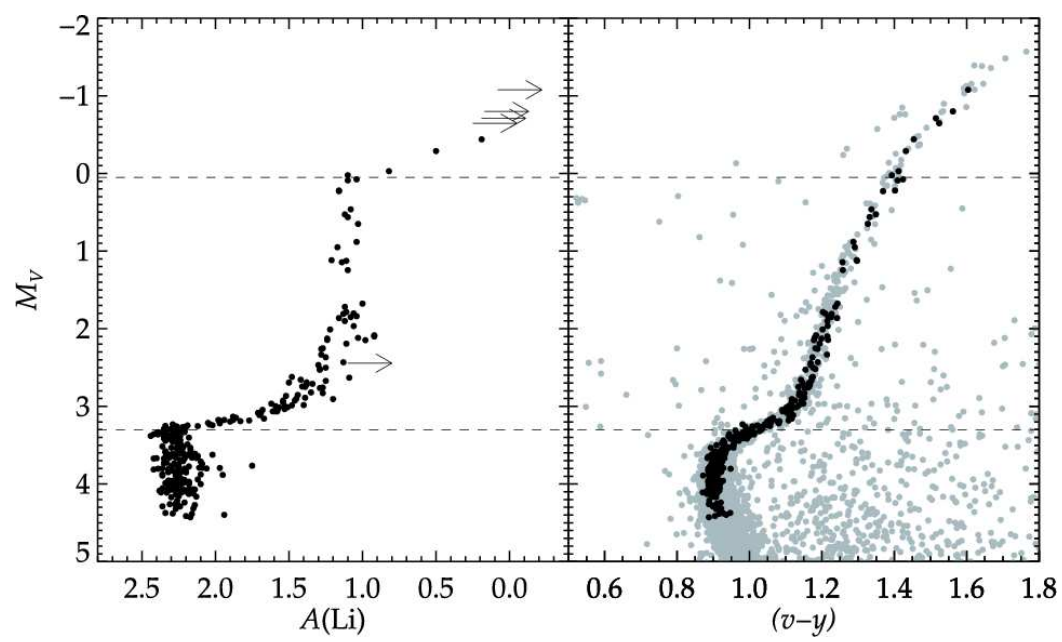

Figure 2: Lithium abundance (from non-LTE analysis) vs. absolute visual magnitude (left) and spectroscopic targets superimposed onto the colour-magnitude diagram (right). [Credit: Lind et al., A\&A, 503, 545, 2009, reproduced with permission (C) ESO.]

ii. Measurements of carbon and nitrogen in globular clusters have enabled quantitative tests of stellar evolution models that include thermohaline mixing [8]. (See Figure 3.)

iii. Building upon earlier work by [9] and [10], there is now compelling evidence that every well studied Galactic globular cluster shows a large spread in the abundances of oxygen and sodium including a prominent O-Na anticorrelation [11] (see Figure 4). Such abundance patterns are believed to be the result of hydrogen-burning at high temperature. With the discovery of these light element abundance variations in unevolved stars [12], the consensus view is that these abundance variations have been imprinted on globular cluster stars from their birth rather than being the product of nucleosynthesis and mixing within the observed stars. Intermediate-mass $\left(4 \mathrm{M}_{\odot}<\mathrm{M}\right.$ $<8 \mathrm{M}_{\odot}$ ) asymptotic giant branch (AGB) stars are a leading candidate for producing the light element abundance variations. In this context, [13] suggested that the most extreme values (O-poor, Na-rich) can be produced in AGB stars only if the [14] rate for the ${ }^{23} \mathrm{Na}(\mathrm{p}, \alpha){ }^{20} \mathrm{Ne}$ reaction is lowered by a factor of four (see Figure 5).

$i v$. The abundances of magnesium and aluminium present, in some globular clusters, a star-tostar variation and anticorrelation [15]. As discussed by [16], the presence of a $\mathrm{Mg}$ - $\mathrm{Al}$ anticorrelation may be related to a combination of mass and metallicity in the cluster. Fast rotating massive stars have been suggested as an alternative site to AGB stars for producing the light element abun- 


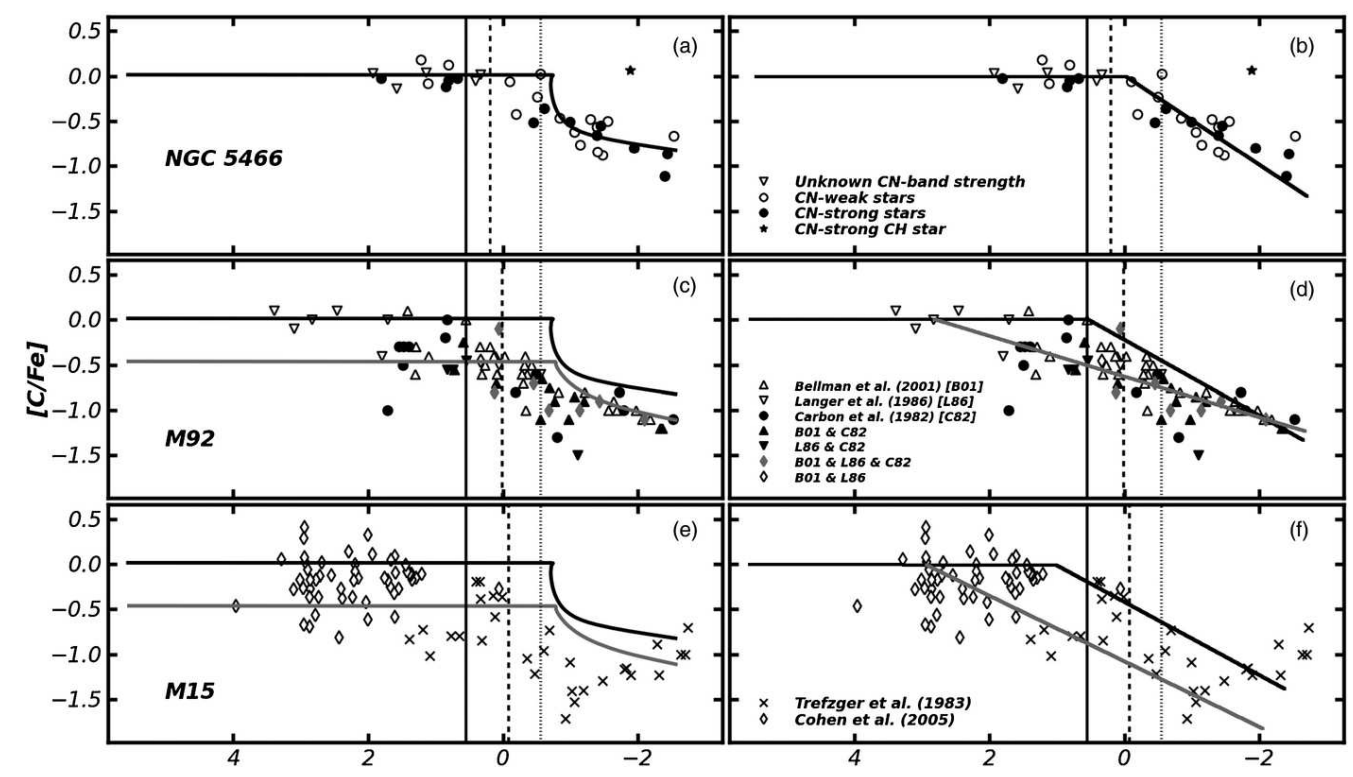

Figure 3: $[\mathrm{C} / \mathrm{Fe}]$ vs. $M_{V}$ for NGC 5466, M92, and M15. (Taken from Figure 2, The Role of Thermohaline Mixing in Intermediate- and Low-metallicity Globular Clusters, Angelou et al., The Astrophysical Journal, Volume 749, Issue 2, 2012, p 128 [8]. Reproduced by permission of the AAS.)

dance variations. For massive stars to produce the observed $\mathrm{Mg}-\mathrm{Al}$ anticorrelation, [17] find that the [18] rate for the ${ }^{24} \mathrm{Mg}(\mathrm{p}, \gamma){ }^{20} \mathrm{Ne}$ reaction needs to be increased by a factor of 1000 at $50 \times$ $10^{6} \mathrm{~K}$.

v. Measurements of the neutron-capture elements in globular clusters have provided unique observational insight into the $s$-process at low metallicity. In the globular cluster $\mathrm{M} 22,[\mathrm{Fe} / \mathrm{H}] \simeq$ -1.7, [19] made an empirical estimate of the $s$-process "residual" by comparing two populations of stars, one with a $r$-process only element abundance pattern and the other with an $r+s$-process element abundance pattern. They find that the $s$-process "residual" cannot be explained by AGB stars with masses $\leq 3 \mathrm{M}_{\odot}$. In their analysis, [19] considered the more metal-rich pair of globular clusters $\mathrm{M} 4$ and $\mathrm{M} 5,[\mathrm{Fe} / \mathrm{H}] \simeq-1.2$. When subtracting the heavy element abundances of M5 from those of M4 (as published by [20]), [19] find that the $s$-process residual is remarkably similar to that of M22. (See the contribution in these proceedings "The s-process in globular cluster M22: hints for higher-mass polluters" by Roederer.)

\section{Multiple Populations}

The discovery of complex structure in colour-magnitude diagrams demonstrates that globular clusters harbour discrete, multiple populations with distinct ages and/or chemical compositions [21]. When using appropriate photometric filters, it is possible to identify stars lying on various parts of the O-Na abundance distribution [22-24].

In Figure 6, three clusters with differing degrees of photometric and chemical diversity are shown. The simplest cluster is M4 in which there is a single metallicity, a single O-Na anticorrelation and one value for the $s$-process ratio $[\mathrm{Ba} / \mathrm{Fe}]$. Our current understanding is that the majority of globular clusters behave like M4, although there are many outstanding issues concerning the 


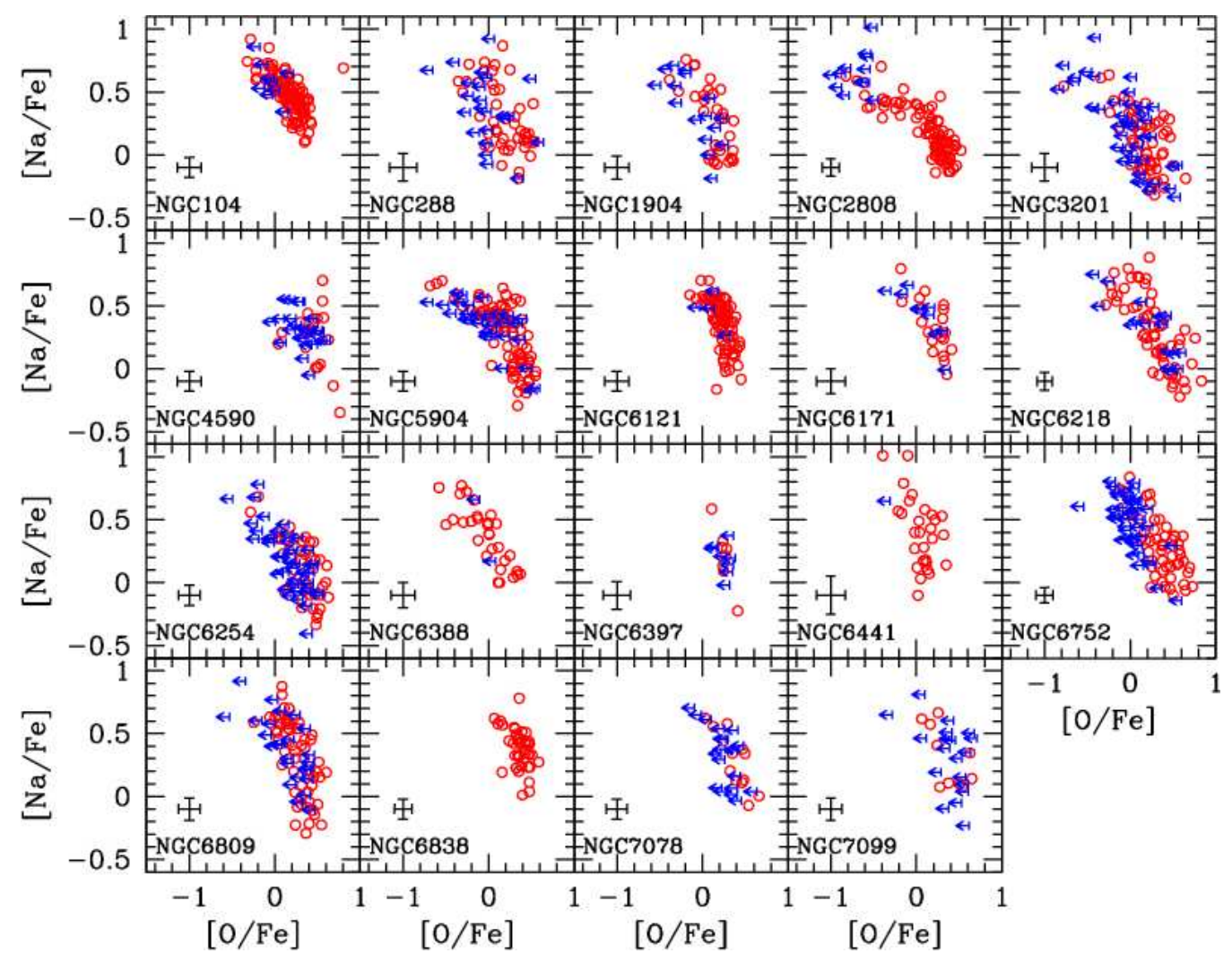

Figure 4: The O-Na anticorrelation for 19 globular clusters. [Credit: Carretta et al., A\&A, 505, 117, 2009, reproduced with permission (C) ESO.]

O-Na anticorrelation including (i) the nature of the polluters, (ii) the mass range involved, (iii) the enrichment timescales, (iv) the relationship with global parameters (mass, HB morphology, etc.) and (v) possible differences with environment, e.g., Milky Way vs. Magellanic Clouds, [6]. M22 is a more complex system in which there is a bimodal metallicity distribution function, two O-Na anticorrelations and a large spread in the $s$-process ratio $[\mathrm{La} / \mathrm{Fe}]$. Additionally, M22 shows a double subgiant branch, a feature also shared by other globular clusters [27]. Finally, $\omega$ Centauri has long been regarded as the most enigmatic of the globular clusters with a significant spread in metallicity. It also exhibits a large range in $\mathrm{O}-\mathrm{Na}$ abundances as well as a distinct pattern in the $[\mathrm{La} / \mathrm{Fe}]$ vs. $[\mathrm{Fe} / \mathrm{H}]$ plane.

\section{Future Directions}

Understanding the chemical enrichment histories of all clusters, from the least to the most complex, represents one of the major challenges in modern astronomy. There are a number of areas in which progress can be made.

NUCLEOSYNTHESIS: None of the candidate polluters can successfully reproduce the observed abundance patterns [6]. Additional measurements of fluorine abundances and isotope ratios of $\mathrm{Mg}$ will be of great value (see the contribution in these proceedings "A different view on light element anticorrelations in globular clusters: fluorine variations in the globular cluster NGC6656(M22)" 


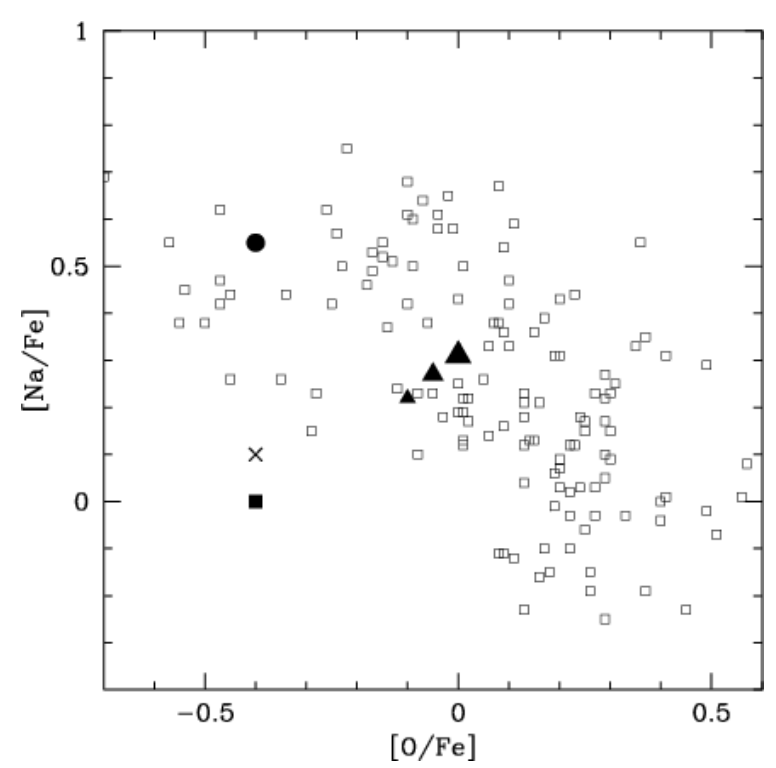

Figure 5: Yields from AGB models with different assumptions concerning the cross sections of the main channel for sodium destruction (full square, cross, and full circle). Measurements in globular clusters are shown as open squares. Triangles represent yields from models calculated with extra mixing. [Credit: Ventura \& D’Antona, A\&A, 457, 995, 2006, reproduced with permission (C) ESO.]
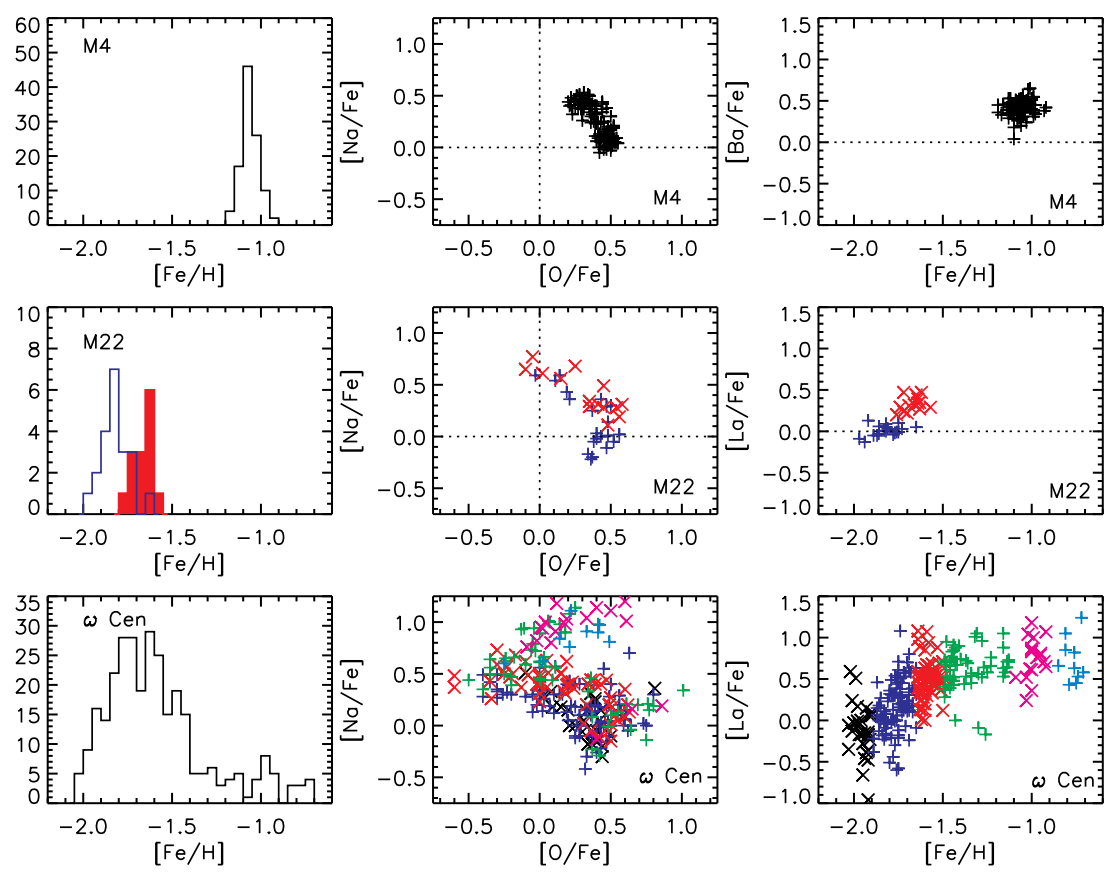

Figure 6: The metallicity distribution functions (left), [Na/Fe] vs. $[\mathrm{O} / \mathrm{Fe}]$ (right) and $[\mathrm{Ba}, \mathrm{La} / \mathrm{Fe}]$ vs. $[\mathrm{Fe} / \mathrm{H}]$ (right) for the globular clusters M4 (upper), M22 (middle) and $\omega$ Cen (lower). Data are taken from [23, 25, 26].

by D'Orazi et al.). Mg represents one of the few elements for which isotope ratios can be measured in globular clusters [15]. Isotope ratios offer a more direct probe of the nucleosynthetic 
processes responsible for the light element abundance variations. In the handful of globular clusters in which fluorine has been measured [28 -30], there is a large star-to-star abundance variations. Thus, fluorine offers new insight into the light element abundance variations in globular clusters and potentially a new check on nuclear reaction rates.

Chemo-dynamical modelling: A physical understanding of the processes which led to the formation of multiple populations in globular clusters likely awaits detailed chemo-dynamical models. While a number of efforts have already been conducted [31, 32], additional work is strongly encouraged.

Given how quickly this field is moving, it will be of great interest to re-assess our understanding of chemical abundances in globular clusters at the next Nuclei in the Cosmos meeting.

\section{Questions}

S. Martell: You showed a correlation between $\mathrm{Nd}$ and $\mathrm{Ni}$ abundance. There are a lot of neutron-capture elements: in measuring $\mathrm{Nd}$, were you lucky to find the one element that's interesting or is there a larger connection between neutron-capture elements and iron-peak elements?

D. Yong: The Nd-Ni correlation is seen in some preliminary work on high precision chemical abundance analyses. In that work, we find a correlation between every combination of elements including alpha, Fe-peak and neutron-capture species. We are still working on the interpretation.

M. Lugaro: Are s-process elements correlated with $\mathrm{O}$ and $\mathrm{Na}$ ? (e.g. the $[\mathrm{La} / \mathrm{Fe}]$ in $\mathrm{M} 22$ )

D. Yong: There are a small number of clusters (including NGC 1851, NGC 6752) in which there appear to be correlations between the light elements (e.g., $\mathrm{O}$ and $\mathrm{Na}$ ) and the s-process elements.

\section{Acknowledgments}

I would like to thank Jay Anderson and Anna Marino for providing material for the presentation and Amanda Karakas for helpful comments. Additionally, I would like to thank the International Advisory Committee and Local Organising Committee for their efforts in organising this successful conference.

\section{References}

[1] Renzini, A., \& Buzzoni, A. Global properties of stellar populations and the spectral evolution of galaxies. Spectral Evolution of Galaxies, 122, 195, 1986

[2] Grundahl, F., VandenBerg, D. A., Bell, R. A., Andersen, M. I., \& Stetson, P. B. A Distance-Independent Age for the Globular Cluster M92. AJ, 120, 1884, 2000

[3] Bennett, C. L., Halpern, M., Hinshaw, G., et al. First-Year Wilkinson Microwave Anisotropy Probe (WMAP) Observations: Preliminary Maps and Basic Results. ApJS, 148, 1, 2003

[4] Kraft, R. P. Abundance differences among globular-cluster giants: Primordial versus evolutionary scenarios. PASP, 106, 553, 1994 
[5] Gratton, R., Sneden, C., \& Carretta, E. Abundance Variations Within Globular Clusters. ARA\&A, 42, 385,2004

[6] Gratton, R., Carretta, E., \& Bragaglia, A. Multiple populations in globular clusters. Lessons learned from the Milky Way globular clusters. A\&ARv, 20, 50, 2012

[7] Lind, K., Primas, F., Charbonnel, C., Grundahl, F., \& Asplund, M. Signatures of intrinsic Li depletion and Li-Na anti-correlation in the metal-poor globular cluster NGC 6397. A\&A 503, 545, 2009

[8] Angelou, G. C., Stancliffe, R. J., Church, R. P., Lattanzio, J. C., \& Smith, G. H. The Role of Thermohaline Mixing in Intermediate- and Low-metallicity Globular Clusters. ApJ, 749, 128, 2012

[9] Sneden, C., Kraft, R. P., Prosser, C. F., \& Langer, G. E. Oxygen abundances in halo giants. I - Giants in the very metal-poor globular clusters M92 and M15 and the metal-poor halo field. AJ, 102, 2001, 1991

[10] Kraft, R. P., Sneden, C., Langer, G. E., \& Prosser, C. F. Oxygen abundances in halo giants. II - Giants in the globular clusters M13 and M3 and the intermediately metal-poor halo field. AJ, 104, 645, 1992

[11] Carretta, E., Bragaglia, A., Gratton, R. G., et al. Na-O anticorrelation and HB. VII. The chemical composition of first and second-generation stars in 15 globular clusters from GIRAFFE spectra. $A \& A, \mathbf{5 0 5}, 117,2009$

[12] Gratton, R. G., Bonifacio, P., Bragaglia, A., et al. The O-Na and Mg-Al anticorrelations in turn-off and early subgiants in globular clusters. A\&A, 369, 87, 2001

[13] Ventura, P., \& D’Antona, F. Does the oxygen-sodium anticorrelation in globular clusters require a lowering of the ${ }^{23} \mathrm{Na}(p, \alpha)^{20} \mathrm{Ne}$ reaction rate? A\&A, 457, 995, 2006

[14] Hale, S. E., Champagne, A. E., Iliadis, C., et al. Investigation of the ${ }^{23} \mathrm{Na}(p, \gamma){ }^{24} \mathrm{Mg}$ and ${ }^{23} \mathrm{Na}(p, \alpha)$ ${ }^{20}$ Ne reactions via $\left({ }^{3} \mathrm{He}, d\right)$ spectroscopy. Phys. Rev. C, 70, 045802, 2004

[15] Yong, D., Grundahl, F., Lambert, D. L., Nissen, P. E., \& Shetrone, M. D. Mg isotopic ratios in giant stars of the globular cluster NGC 6752. A\&A, 402, 985, 2003

[16] Carretta, E., Bragaglia, A., Gratton, R. G., et al. Properties of stellar generations in globular clusters and relations with global parameters. A\&A, 516, 55, 2010

[17] Decressin, T., Meynet, G., Charbonnel, C., Prantzos, N., \& Ekström, S. Fast rotating massive stars and the origin of the abundance patterns in galactic globular clusters. A\&A, 464, 1029, 2007

[18] Iliadis, C., D’Auria, J. M., Starrfield, S., Thompson, W. J., \& Wiescher, M. Proton-induced Thermonuclear Reaction Rates for A=20-40 Nuclei. ApJS 134, 151, 2001

[19] Roederer, I. U., Marino, A. F., \& Sneden, C. Characterizing the Heavy Elements in Globular Cluster M22 and an Empirical s-process Abundance Distribution Derived from the Two Stellar Groups. ApJ, 742, 37, 2011

[20] Yong, D., Karakas, A. I., Lambert, D. L., Chieffi, A., \& Limongi, M. Heavy Element Abundances in Giant Stars of the Globular Clusters M4 and M5. ApJ, 689, 1031, 2008

[21] Milone, A. P., Bedin, L. R., Piotto, G., et al. The ACS Survey of Galactic Globular Clusters. III. The Double Subgiant Branch of NGC 1851. ApJ, 673, 241, 2008

[22] Yong, D., Grundahl, F., Johnson, J. A., \& Asplund, M. Nitrogen Abundances in Giant Stars of the Globular Cluster NGC 6752. ApJ, 684, 1159, 2008

[23] Marino, A. F., Villanova, S., Piotto, G., et al. Spectroscopic and photometric evidence of two stellar populations in the Galactic globular cluster NGC 6121 (M 4). A\&A, 490, 625, 2008 
[24] Milone, A. P., Piotto, G., Bedin, L. R., et al. Multiple Stellar Populations in 47 Tucanae. ApJ, 744, 58, 2012

[25] Marino, A. F., Milone, A. P., Piotto, G., et al. A double stellar generation in the globular cluster NGC 6656 (M 22). Two stellar groups with different iron and s-process element abundances. A\&A, $\mathbf{5 0 5}$, 1099, 2009

[26] Marino, A. F., Milone, A. P., Piotto, G., et al. Sodium-Oxygen Anticorrelation and Neutron-capture Elements in Omega Centauri Stellar Populations. ApJ, 731, 64, 2011

[27] Piotto, G., Milone, A. P., Anderson, J., et al. Hubble Space Telescope reveals multiple Sub-Giant Branch in eight Globular Clusters. ApJ in press, arXiv:1208.1873

[28] Smith, V. V., Cunha, K., Ivans, I. I., et al. Fluorine Abundance Variations in Red Giants of the Globular Cluster M4 and Early-Cluster Chemical Pollution. ApJ, 633, 392, 2005

[29] Yong, D., Meléndez, J., Cunha, K., et al. Chemical Abundances in Giants Stars of the Tidally Disrupted Globular Cluster NGC 6712 from High-resolution Infrared Spectroscopy. ApJ 689, 1020, 2008

[30] Alves-Brito, A., Yong, D., Meléndez, J., Vásquez, S., \& Karakas, A. I. CNO and $F$ abundances in the globular cluster M 22 (NGC 6656). A\&A 540, A3, 2012

[31] D’Ercole, A., Vesperini, E., D’Antona, F., McMillan, S. L. W., \& Recchi, S. Formation and dynamical evolution of multiple stellar generations in globular clusters. MNRAS, 391, 825, 2008

[32] Bekki, K., \& Yong, D. On the origin of the stellar halo and multiple stellar populations in the globular cluster NGC 1851. MNRAS, 419, 2063, 2012 